\title{
Analytic approach to the complete set of QED corrections to fermion pair production in $\mathrm{e}^{+} \mathrm{e}^{-}$- annihilation
}

\author{
D. Bardin, M. Bilenky, A. Chizhov, A. Sazonov \\ Joint Institute for Nuclear Research, Dubna, \\ Head Post Office P.O. Box 79, SU - 101000 Moscow, USSR \\ O. Fedorenko \\ Petrosavodsk State University, SU-185018 Petrosavodsk, USSR \\ and \\ T. Riemann, M. Sachwitz \\ Institut für Hochenergiephysik, \\ Platanenallee 6, DDR-1615 Zeuthen/Brandenburg*
}

18 March 1990

\begin{abstract}
We present the convolution integral for fermion pair production in the electroweak standard theory to order $\mathrm{O}(\alpha)$ including also soft photon exponentiation. The result is complete in the sense that it includes initial and final state radiation and their interference. From the basic result analytic formulae for the differential cross section - we also derive the corresponding expressions for total cross section $\sigma_{T}$ and integrated forward-backward asymmetry $A_{F B}$. The numerical importance of different contributions for the analysis of experiments at LEP/SLC energies is discussed.
\end{abstract}

*Address to be expected after 3 October 1990: O-1615 Zeuthen/Brandenburg(FRG) 
1. Introduction (3)

2. Notations and phase space parametrisation (3)

3. Initial state radiation (7)

3.1. The radiator functions for the angular distribution (7)

3.2. Soft photon exponentiation (9)

3.3. Integrated cross section and asymmetry (9)

4. Initial-final state interference radiation (13)

4.1. The radiator functions for the angular distribution (13)

4.2. Integrated cross section and asymmetry (16)

5. Final state radiation (19)

5.1. The radiator functions for the angular distribution (19)

5.2 . Integrated cross section and asymmetry to order $\mathrm{O}(\alpha)(20)$

5.3 Soft photon exponentiation (20)

6. Discussion (22) 


\section{INTRODUCTION}

Stimulated by the possibility of very precise experiments at $\mathrm{e}^{+} \mathrm{e}^{-}$- storage rings, the QED corrections for the reaction

$$
e^{+}\left(k_{2}\right)+e^{-}\left(k_{1}\right) \longrightarrow(\gamma, Z) \longrightarrow f^{+}\left(p_{2}\right)+f^{-}\left(p_{1}\right)+n \gamma(p)
$$

have been studied in very detail for a large energy region including the $\mathrm{Z}$ resonance. A comprehensive collection and comparison of available results within the electroweak standard theory $\mathbb{}$ may be found in and in references quoted therein. Analytic and semi-analytic formulae proved to be of great value both for a deeper understanding of process ( 1) and for ensuring a high reliability of Monte Carlo codes 3. Recently, the analytic approach to QED has also been used for the analysis of $\mathrm{Z}$ line shape data at LEP/SLC 4 . The line shape is the integrated total cross section $\sigma_{T}(\mathrm{~s})$ as function of $s=4 E^{2}$ where $E$ is the beam energy. This most inclusive observable has been studied theoretically since many years and is well-described to order $\mathrm{O}(\alpha)-10$ with soft photon exponentiation [10, 11] and also for several massive intermediate bosons 12, higher order leading logarithmic corrections [13, 14 and the complete QED initial state radiation corrections to order $\mathrm{O}\left(\alpha^{2}\right)$ 15. A compilation of many important contributions is contained in 16 .

Only recently, similar analytic results have been published for the integrated forward-backward asymmetry $A_{F B}$. The complete $\mathrm{O}(\alpha)$ convolution integral with soft photon exponentiation for $A_{F B}$ was obtained in [17] (see also 8 ) and the leading logarithmic approximation for initial state radiation including $\mathrm{O}\left(\alpha^{2}\right)$ terms in 18. A survey on results about $A_{F B}$ is contained in 19.

Compared to $\sigma_{T}$ and $A_{F B}$, analytic results for the differential cross section $\mathrm{d} \sigma / \mathrm{d} \cos \Theta$ are scarce. Earlier attempts are 20, 21. Other distributions are treated in 22. In pure QED, the first compact analytic expressions for hard bremsstrahlung corrections to the differential cross section have been derived in 23. A formalism for leading logarithmic approximations may be found in 18, though without application to $\mathrm{d} \sigma / \mathrm{d} \cos \Theta$. Recently, semi-analytic formulae allowing for quite realistic cuts have been applied in 24. This article contains the systematic presentation of an analytic calculation of QED corrections to the angular distribution for reaction (1). Though computer codes relying on the present results have already been released and used f25 for applications in LEP physics, very few of the material has been presented so far 26]; see also [3, 16, 19. In chapter 2 we introduce the notation and describe in short two derivations of the analytic expressions for the angular distribution. Chapter 3 to 5 contain the basic result which consists of compact, explicit expressions for the soft and hard photon radiator functions in the convolution integral due to initial (chapter 3) and final (chapter 5) state radiation and their interference (chapter 4) to order $\mathrm{O}(\alpha)$. They also include higher order corrections and some formulae for $\sigma_{T}$ and $A_{F B}$. Chapter 6 contains numerical results and conclusions.

\section{NOTATIONS AND PHASE SPACE PARAMETRISATION}

The explicit analytic formulae for the differential cross section proved to be too cumbersome to be described in detail here. For that reason, we choose a semi- analytic presentation with the 
following notation:

$$
\frac{d \sigma}{d c}=\sum_{m, n=0,1} \sum_{A=T, F B} \sum_{a=e, i, f} \operatorname{Re}\left[\sigma_{A}^{a, 0}(s, s ; m, n) R_{A}^{a}(c ; m, n)\right] .
$$

The scattering angle $c=\cos \Theta$ is defined between the produced fermion $f^{+}(f=\mu, \nu, q)$ and the positron beam. The functions $\sigma_{A}^{a, 0}(s, s ; m, n)$ are reduced Born cross sections defined below and the corrections $R_{A}^{a}(c ; m, n)$ can be expressed by semi-analytic formulae for soft and hard parts of QED bremsstrahlung radiator functions $S_{A}^{a}(c, \epsilon ; m, n)$ and $H_{A}^{a}(v, c)$ :

$$
R_{A}^{a}(c ; m, n)=\int_{0}^{\Delta} d v \frac{\sigma_{A}^{a, 0}\left(s, s^{\prime} ; m, n\right)}{\sigma_{A}^{a, 0}(s, s ; m, n)}\left[\delta(v) S_{A}^{a}(c, \epsilon ; m, n)+\theta(v-\epsilon) H_{A}^{a}(v, c)\right] .
$$

In (3), we allow for a possible cut-off $\Delta\left(0<\Delta \leq 1-4 \mathrm{~m}_{f}^{2} / \mathrm{s}\right)$ on the energy $v$ of the emitted photon in units of the beam energy. The effective energy $s^{\prime}$ of the created fermion pair is $s^{\prime}=(1-v) s$. The sums over $m, n$ in ( 2) include photon $(m=0)$ and $\mathrm{Z}$ boson $(m=1)$ exchange. A generalisation to the case of additional vector bosons $\mathrm{Z}_{n}, n>1$, is straightforward. Indices $a=e, f, i$ are used for initial and final state radiation and their interference. The angular dependence of the Born cross section has been formally included into the initial state contribution $a=e$. The P-even and P-odd cross section parts carry index $A=T$ and $A=F B$. Under CP-invariance, they are also C-even (C-odd), correspondingly . After symmetric (anti-symmetric) integration over $\cos \Theta$ the $R_{T^{-}}^{a}$ functions ( $R_{F B^{-}}^{a}$ functions) yield the total cross section $\sigma_{T}$ (the integrated forward-backward asymmetry $\left.A_{F B}\right)$ :

$$
\begin{gathered}
\sigma_{T}=\int_{-1}^{1} d c \frac{d \sigma}{d c} \\
A_{F B}=\frac{\sigma_{F B}}{\sigma_{T}}=\frac{1}{\sigma_{T}}\left[\int_{0}^{1} d c \frac{d \sigma}{d c}-\int_{-1}^{0} d c \frac{d \sigma}{d c}\right] .
\end{gathered}
$$

Within our terminology,

$$
\begin{gathered}
\sigma_{A}=\sum_{m, n=0,1} \sum_{a=e, i, f} \operatorname{Re}\left[d_{A} \sigma_{A}^{a, 0}(s, s ; m, n) R_{A}^{a}(m, n)\right], \\
R_{A}^{a}(m, n)=d_{A}^{-1} \int_{0}^{1} d c R_{A}^{a}(c ; m, n), \quad A=T, F B, \\
d_{T}=\frac{4}{3}, \quad d_{F B}=1 .
\end{gathered}
$$

The additional numerical factors in ( 6, 7) ensure the usual total cross section normalisations for $\sigma_{T}$ and $\sigma_{F B}$.

The dependence of $\mathrm{d} \sigma / \mathrm{d} c$ on weak neutral couplings and electric charges of the fermions and on possible polarisations and weak loop corrections together with the typical Born-like resonance behaviour have been collected in the explicit Born factors $\sigma_{A}^{a, 0}(s, s ; m, n)$. As a consequence, the functions $R_{A}^{a}(c ; m, n)$ depend only on the $\mathrm{Z}$ boson mass $M_{Z}=M_{1}$ and width $\Gamma_{Z}=\Gamma_{1}$ 27, 16] and on kinematic variables $s, \cos \Theta$, and the energy cut-off $\Delta$. Of course, fermion masses which are assumed here to be small compared to $s, M_{Z}, \Gamma_{Z}$ show up in certain logarithmic mass singularities.

The reduced Born cross sections $\sigma_{A}^{a, 0}\left(s, s^{\prime} ; m, n\right)$ have different energy dependence for $a=e, i, f$ :

$$
\begin{aligned}
\sigma_{A}^{e, 0}\left(s, s^{\prime} ; m, n\right) & =\sigma_{A}^{0}\left(s^{\prime}, s^{\prime} ; m, n\right), \\
\sigma_{A}^{i, 0}\left(s, s^{\prime} ; m, n\right) & =\sigma_{\bar{A}}^{0}\left(s, s^{\prime} ; m, n\right),
\end{aligned}
$$




$$
\sigma_{A}^{f, 0}\left(s, s^{\prime} ; m, n\right)=\sigma_{A}^{0}(s, s ; m, n) .
$$

Here, $\bar{A}=F B$ or $T$ if $A=T$ or $F B$, and

$$
\begin{gathered}
\sigma_{A}^{0}\left(s, s^{\prime} ; m, n\right)=\frac{\pi \alpha^{2}}{2 s} C_{A}\left(m, n ; \lambda_{1}, \lambda_{2}, h_{1}, h_{2}\right) c_{f} \frac{1}{2}\left[\chi_{m}\left(s^{\prime}\right) \chi_{n}^{*}(s)+\chi_{m}(s) \chi_{n}^{*}\left(s^{\prime}\right)\right], \\
\chi_{n}(s)=\frac{g_{n}^{2}}{4 \pi \alpha} \frac{s}{s-m_{n}^{2}}, \\
m_{n}^{2}=M_{n}^{2}-i M_{n} \Gamma_{n}(s), \\
\Gamma_{n}(s) \simeq \frac{s}{M_{n}^{2}} \Gamma_{n} .
\end{gathered}
$$

In ( 12), the fermions have a color factor $c_{f}=3$ in case of quarks and $c_{f}=1$ for leptons. Their vector and axial vector couplings $v_{f}(n)$ and $a_{f}(n)$ to gauge boson $\mathrm{Z}_{n}$ are contained in $C_{A}$ :

$$
\begin{aligned}
& C_{T}\left(m, n ; \lambda_{1}, \lambda_{2}, h_{1}, h_{2}\right)= \\
& \left\{\lambda_{1}\left[v_{e}(m) v_{e}^{*}(n)+a_{e}(m) a_{e}^{*}(n)\right]+\lambda_{2}\left[v_{e}(m) a_{e}^{*}(n)+v_{e}^{*}(n) a_{e}(m)\right]\right\} \\
& \left\{h_{1}\left[v_{f}(m) v_{f}^{*}(n)+a_{f}(m) a_{f}^{*}(n)\right]+h_{2}\left[v_{f}(m) a_{f}^{*}(n)+v_{f}^{*}(n) a_{f}(m)\right]\right\}, \\
& C_{F B}\left(m, n ; \lambda_{1}, \lambda_{2}, h_{1}, h_{2}\right)=C_{T}\left(m, n ; \lambda_{2}, \lambda_{1}, h_{2}, h_{1}\right) .
\end{aligned}
$$

We allow for longitudinal polarisations $\lambda_{-}, \lambda_{+}$of both the electron and positron beams and for final states with helicities $h_{-}, h_{+}$. Due to the CP-invariance of the problem and the $(v, a)$ structure of the interactions, the following combinations of polarisations are possible:

$$
\begin{aligned}
\lambda_{1}=1-\lambda_{+} \lambda_{-}, & \lambda_{2}=\lambda_{+}-\lambda_{-}, \\
h_{1}=\frac{1}{4}\left(1-h_{+} h_{-}\right), & h_{2}=\frac{1}{4}\left(h_{+}-h_{-}\right) .
\end{aligned}
$$

In case of the standard theory 11 , we use the conventional couplings of photon and $\mathrm{Z}$ boson:

$$
\begin{gathered}
g_{0}=e, \quad v_{f}(0)=Q_{f}, \quad a_{f}(0)=0, \\
g_{1}=\left(\sqrt{2} G_{\mu} M_{Z}^{2}\right)^{1 / 2}, \quad v_{f}(1)=I_{3}^{L}(f)-2 Q_{f} \sin ^{2} \Theta_{W}, \quad a_{f}(1)=I_{3}^{L}(f),
\end{gathered}
$$

where $I_{3}^{L}(f)$ is the third component of the weak isospin of fermion $f ; 2 I_{3}^{L}(e)=Q_{e}=-1$.

The inclusion of non-QED weak loop effects (see 28, 29, 30, 20 and references quoted therein) is trivial if one uses the form factor approach for their description 29. Coupling constants $g_{0}, g_{1}$, and the vector couplings $v_{e}(1), v_{f}(1)$ become complex and $s$-dependent. Further details are explained in 30, 19. For an exact treatment, an additional form factor $v_{e f}(1)$ replaces the product $v_{e}(1) v_{f}(1)$ in (16) whenever it appears there. In principle, energy-dependent form factors should be understood to be part of the integrand in (2, 3). We have checked by explicit comparisons that, due to the minor s-dependence of them, the numerical error which is implied by the approximation is practically negligible [31, 3] .

For notational convenience, all angular dependences have been included into the radiator functions. The functions $R_{A}^{a}(c ; m, n)$ of (3) are result of an incoherent sum of real and virtual photonic corrections. They are obtained by straightforward but lengthy Feynman diagram calculations with extensive use of the analytic manipulation programs SCHOONSCHIP 32 and REDUCE 33 . The $R_{A}^{a}(c ; m, n)$ are gauge-invariant and, if integrated without cuts, also Lorentz-invariant. Determinations of the vertex and box corrections 34, soft photon and hard photon contributions deserve different techniques, correspondingly. 
We would like to give short comments on the two methods which have been used for the $\mathrm{O}(\alpha)$ bremsstrahlung phase space integrations. In one approach, we used the so called $R_{\gamma}$ system, the rest system of $\left(f^{-} \gamma\right)$, where the three-momentum relation $\vec{p}\left(f^{-}\right)+\vec{p}(\gamma)=0$ is fulfilled. The phase space parametrisation chosen is:

$$
\int d \Gamma=\frac{\pi^{2}}{4 s^{3}} \int_{-c_{m}}^{c_{m}} d \cos \Theta \int_{0}^{s} x d x \frac{s-x}{s-x+m_{f}^{2}} \frac{1}{4 \pi} \int_{-1}^{1} d \cos \Theta_{\gamma}^{R} \int_{0}^{2 \pi} d \varphi_{\gamma}^{R},
$$

where $\left(\Theta_{\gamma}^{R}, \varphi_{\gamma}^{R}\right)$ are photon angles in the $R_{\gamma}$ - system and $x$ the momentum fraction of $f^{+}$in the cms in units of the beam energy:

$$
x=-2 p\left(f^{+}\right)\left[p\left(e^{+}\right)+p\left(e^{-}\right)\right]=2 \sqrt{s} E\left(f^{+}\right) .
$$

Some details of the calculation have been described in 23 , including the n-dimensional treatment of soft photon contributions which follows the methods developed in 35. The hard photon integration has been done with SCHOONSCHIP using tables of integrals 36. The total cross section $\sigma_{T}$ and the integrated forward-backward asymmetry without cut 8 have been determined with this technique as well as the angular distribution which remained unpublished so far with exclusion of the pure QED case 23. Another set of integration variables allows for a cut on the photon energy:

$$
\begin{gathered}
\int d \Gamma=\frac{\pi^{2}}{4 s} \int_{-c_{m}}^{c_{m}} d \cos \Theta \int_{0}^{v_{m}} d v \int_{v_{2, \min }}^{v_{2, \max }} d v_{2} \frac{1}{2 \pi} \int_{0}^{2 \pi} d \varphi_{\gamma} \\
v_{2, \max (\min )}=\frac{1}{2} v\left[1 \pm v_{m}\left(s^{\prime}\right)^{1 / 2}\right] \\
v_{m}(s)=1-4 m_{f}^{2} / s
\end{gathered}
$$

In (24), $\varphi_{\gamma}$ is the azimuthal angle of the photon in the cms and

$$
v_{2}=-\frac{2}{s} p\left(f^{+}\right) p(\gamma)=1-2 E\left(f^{-}\right) / \sqrt{s}
$$

We should also comment on the integration boundaries for $\cos \Theta$ :

$$
c_{m}=1-2 m_{e}^{2} / s
$$

The radiators for the angular distribution contain terms behaving in the hard photon corner like $\ln (1 \pm c),(1 \pm c)^{-2}$ etc. which become singular and for initial state radiation even non-integrable at $c= \pm 1$. In fact these quantities are related to $t=-2 p\left(e^{+}\right) p\left(f^{+}\right)$and $x$ :

$$
\begin{gathered}
t=\frac{x}{2}-\frac{1}{2 s} \sqrt{s^{2}-4 m_{e}^{2} s} \sqrt{x^{2}-4 m_{f}^{2} s} \cos \Theta, \\
t \approx \frac{x}{2}\left[1-\left(1-2 m_{e}^{2} / s\right) \cos \Theta\right] .
\end{gathered}
$$

From (28), it becomes evident that the phase space integrals (22,24) are properly regulated. In order to get a convolution representation for $\mathrm{d} \sigma / \mathrm{d} \cos \Theta$ one has to perform in (24) two integrations. This has been done with SCHOONSCHIP and REDUCE, based on tables of integrals for hard bremsstrahlung. The soft photon part is the same as determined earlier; see, e.g., in 610 . While all the hard photon parts of the radiators for the angular distribution remain independent of the type of exchanged gauge boson, this is not the case for the box diagram contributions to the soft photon part of the QED interference corrections as has been indicated in the notation in 
( 3) . After summing up the vertex or box contributions with those due to soft photons, the resulting $S_{A}^{a}(c, \epsilon ; m, n)$ are infra-red finite but yet dependent on the infinitesimal parameter $\epsilon$ which discriminates between soft and hard photons. The integral over the photon energy in ( 3) as a whole is independent of $\epsilon$ as may be seen from the formulae of the following section. Restricting oneself to a semi-analytic (i.e. containing one numerical integration) result for $\mathrm{d} \sigma / \mathrm{d} c$, one can take into account even cuts on the acollinearity and on the fermion energies as has been demonstrated recently in 24. where also ( 24) was the starting point.

\section{INITIAL STATE RADIATION}

In this chapter, we give a systematic presentation of initial state corrections.

\subsection{THE RADIATOR FUNCTIONS FOR THE ANGULAR DISTRIBUTION}

There are two radiator functions for the QED inital state corrections, both of them containing well-known soft photon parts and hard photon parts which will deserve some comments. The soft photon parts including vertex corrections are:

$$
\begin{gathered}
S_{A}^{e}(c, \epsilon ; m, n)=D_{A}(c)\left[1+S\left(\epsilon, \beta_{e}\right)\right], \quad A=T, F B, \\
D_{T}(c)=1+c^{2}, \\
D_{F B}(c)=2 c,
\end{gathered}
$$

where we include in ( 31) also the Born cross section. Further,

$$
\begin{gathered}
S\left(\epsilon, \beta_{e}\right)=\beta_{e}\left(\ln \epsilon+\frac{3}{4}\right)+\frac{\alpha}{\pi} Q_{e}^{2}\left(\frac{\pi^{2}}{3}-\frac{1}{2}\right), \\
\beta_{e}=\frac{2 \alpha}{\pi} Q_{e}^{2}\left(L_{e}-1\right), \quad L_{e}=\ln \frac{s}{m_{e}^{2}} .
\end{gathered}
$$

The hard photon parts are symmetrised for the C-even part $(A=T)$ and anti-symmetrised for the C-odd part $(A=F B)$ :

$$
H_{T, F B}^{e}(v, c)=\frac{\alpha}{\pi} Q_{e}^{2}\left[h_{T, F B}^{e}(v, c) \pm h_{T, F B}^{e}(v,-c)\right] .
$$

As already stated, the hard radiator parts depend on only two variables $v, c$ :

$$
\begin{array}{r}
h_{T}^{e}(v, c)=\frac{z^{2}}{v^{3}}\left[\frac{L_{c}}{\gamma^{2}} r_{2}\left(r_{2}-\frac{2 z}{\gamma} r_{1}+\frac{2 z^{2}}{\gamma^{2}}\right)+\left(-\frac{2}{3 z} r_{4}+\frac{10}{3} r_{2}-4 z\right)+\frac{2}{3 \gamma z}\left(r_{2} r_{3}\right)-\right. \\
\left.-\frac{1}{\gamma^{2}}\left(3 r_{4}+8 z r_{2}+\frac{26}{3} z^{2}\right)+\frac{z}{\gamma^{3}}\left(8 r_{3}+\frac{44}{3} z r_{1}\right)-\frac{z^{2}}{\gamma^{4}}\left(\frac{22}{3} r_{2}+4 z\right)\right], \\
h_{F B}^{e}(v, c)=\frac{z^{2}}{v^{2}}\left[r_{2} \frac{L_{c}}{\gamma^{2}}\left(r_{1}-\frac{2 z}{\gamma}\right)+\frac{2}{\gamma} r_{2}-\frac{4}{\gamma^{2}} r_{1}\left(r_{2}+z\right)+\frac{2 z}{\gamma^{3}}\left(3 r_{2}+2 z\right)\right] .
\end{array}
$$


The following abbreviations are used:

$$
\begin{gathered}
L_{c}=\ln \frac{\gamma^{2}}{z}+L_{e}, \\
z=1-v, \quad r_{n}=1+z^{n}, \\
c_{ \pm}=\frac{1}{2}(1 \pm c), \quad \gamma=c_{+}+z c_{-} .
\end{gathered}
$$

As one should expect, the distributions are singular for $v$ approaching 1 (hard photon emission) and $c$ approaching \pm 1 (collinear radiation). In the soft photon corner $(v \rightarrow \epsilon)$, the hard radiators become singular too:

$$
\lim _{v \rightarrow \epsilon} H_{A}^{e}(v, c)=\frac{\beta_{e}}{v} D_{a}(c)+O(1), \quad A=T, F B
$$

From (42) the necessary compensation of the dependence on the soft photon parameter $\epsilon$ in (34) is evident. Performing the integration over the photon energy variable $v$ leads to analytic expressions for the initial state corrections $R_{A}^{e}(c ; m, n)$ to $\mathrm{d} \sigma / \mathrm{d} c$ as defined in ( 2-3). These integrations are cumbersome but not too complicated. The structure of the integrands $\sigma_{A}^{e, 0}\left(s, s^{\prime} ; m, n\right) H_{A}^{e}(v, c)$ leads to integrals containing rational functions, logarithms and Euler dilogarithms. The interested reader may envisage the Fortran program MUCUTCOS in the package ZBIZON 38 where the corresponding analytic expressions for the angular distribution are coded. We only would like to comment on the origin of the radiative tail within the present formalism. For initial state radiation, it is useful to linearise in $s^{\prime}$ the resonating function ( 12-13),

$$
\frac{1}{s^{\prime}-m_{n}^{2}} \frac{1}{s^{\prime}-m_{p}^{2 *}}=\frac{1}{m_{p}^{2 *}-m_{n}^{2}}\left[\frac{1}{s^{\prime}-m_{p}^{2 *}}-\frac{1}{s^{\prime}-m_{n}^{2}}\right]
$$

if at least one of the interfering gauge bosons is massive. For $n=p$, there arises the factor

$$
\frac{s}{m_{n}^{2 *}-m_{n}^{2}}=-\frac{i}{2} \frac{M_{n}}{\Gamma_{n}} \frac{s}{M_{n}^{2}},
$$

setting the scale of tail effects, $M_{n} / \Gamma_{n}$. The threshold is defined by the onset of influence of the imaginary quantity (44) onto the real type cross section. So, it needs another imaginary quantity which is found from $\left(s^{\prime}-m_{n}^{2}\right)^{-1}$. After integration over the photon energy, functions of the following type arise:

$$
\begin{gathered}
\int_{\epsilon}^{\Delta} d v \frac{1}{1-v-R_{n}}=-\ln \frac{1-R_{n}-\epsilon}{1-R_{n}-\Delta}=-L_{R_{n}}(\Delta) \\
R_{n}=m_{n}^{2} / s
\end{gathered}
$$

If the cut-off $\Delta$ is not as infinitesimal as $\epsilon$ (i.e. if hard photons are radiated), a substantial imaginary part is developed if $s>M_{n}^{2}$.

The photonic case, $n=p=0$, must be treated with care. In order to get the exact hard bremsstrahlung correction for pure photon exchange one must use a reduced Born cross section (12) which includes an additional phase space factor under the integral:

$$
\sigma_{A}^{0}\left(s^{\prime}, s^{\prime} ; 0,0\right) \Rightarrow \sqrt{1-4 m_{f}^{2} / s^{\prime}}\left(1+2 m_{f}^{2} / s^{\prime}\right) \sigma_{A}^{0}\left(s^{\prime}, s^{\prime} ; 0,0\right) .
$$

The additional threshold factor in (47) influences only a minor part of the calculation and it is not too difficult (though lengthy) to take it into account in an analytic calculation. The pure photonic 
corrections to the angular distribution may be found in 23. The function $F_{0}$ defined there in eqs.(5), (10) corresponds to $R_{T}^{e}(c ; 0,0)$ introduced here in ( 3). Interesting enough, in the existing literature there is no explicit mentioning of the correction $R_{F B}^{e}(c ; 0,0)$ or of its integral which contributes to $A_{F B}$ (see below). Since QED corrections due to photon exchange do not contain axial-type couplings, there does not arise any contribution of $R_{F B}^{e}(c ; 0,0)$ to the cross section - if not at least one beam polarisation and one final particle helicity are nonzero; see ( 16 19).

\subsection{SOFT PHOTON EXPONENTIATION}

As may be seen from (42), the hard parts of the radiator functions become singular in the soft photon corner of the phase space. Their divergent part can be combined with the soft photon radiator (31,34) in order to get the lowest order of an infinite sum over soft photon contributions. The treatment can follow exactly the arguments given in 10, 15 for the total cross section. The result is the following modification of the initial state radiative corrections for the angular distribution:

$$
\begin{gathered}
\bar{R}_{A}^{e}(c ; m, n)=\int_{0}^{\Delta} d v \frac{\sigma_{A}^{0}\left(s^{\prime}, s^{\prime} ; m, n\right)}{\sigma_{A}^{0}(s, s ; m, n)}\left\{D_{A}(c)\left[1+\bar{S}\left(\beta_{e}\right)\right] \beta_{e} v^{\beta_{e}-1}+\bar{H}_{A}^{e}(v, c)\right\}, \\
\bar{H}_{A}^{e}(v, c)=H_{A}^{e}(v, c)-\frac{\beta_{e}}{v} D_{A}(c), \\
\bar{S}\left(\beta_{e}\right)=\frac{\alpha}{\pi} Q_{e}^{2}\left[\frac{\pi^{2}}{3}-\frac{1}{2}+\frac{3}{2}\left(L_{e}-1\right)\right] .
\end{gathered}
$$

In fig. 1 the contributions of the initial state radiation corrections to the differential cross section are shown as functions of the scattering angle.

We choose as typical energies $\sqrt{s}=30 \mathrm{GeV}$ (TRISTAN); $91.1 \mathrm{GeV}$ (the $\mathrm{Z}$ boson mass value; LEP,SLC) and $200 \mathrm{GeV}$ (tail region, LEP 200). The relative importance of the cross section contributions depend on three different components; the coupling combinations $C_{A}(m, n)$ which are in addition channel dependent, the factors $\left(1, \chi,|\chi|^{2}\right)$ which have been split away for reasons explained above, and then the Born plus QED correction factors. Shown are the contributions for soft photon exponentiation. At lower energies, pure photonic corrections naturally dominate for $\sigma_{T}$ while they are zero for $\sigma_{F B}$ (if there are no polarisation phenomena). At the $\mathrm{Z}$ peak and beyond in the tail region, the $\mathrm{Z}$ exchange dominates. For $|\cos \Theta|$ approaching 1 (beam directions), the cross section rises extremely due to the collinear hard photon emission. In fig. 2 the net Born plus initial state radiation cross section is shown as function of the scattering angle with the photon energy cut-off $\Delta$ as parameter. In the lower energy range the angular distribution is nearly symmetric due to the dominance of pure QED. The same is true at resonance, but here it is due to the smallness of the coupling combination accompanying the nonsymmetric pure $\mathrm{Z}$ exchange contribution (in case of muon production). In the tail region, a pronounced non-symmetric angular behaviour occurs. Hard bremsstrahlung leads to a rising of the cross section with exclusion of the resonance region where due to initial state radiation the reduced effective energy falls below the peaking value. A cut on the maximal photon energy reduces the cross section while soft photon exponentiation compensates at least partly the negative virtual plus soft photon terms.

\subsection{INTEGRATED CROSS SECTION AND FORWARD BACKWARD ASYMMETRY}


Integrating over the angular dependence of the initial state radiation ( 31, 36), one gets the radiator functions for $\sigma_{T}\left[5\right.$ and $A_{F B}[17$. These are definitely different from each other already in the $\mathrm{O}(\alpha)$ leading logarithmic approximation. To some extent, this remained unclear in the comment on that point in 18. Nevertheless, at the $\mathrm{Z}$ peak the differences are small 42. For more details we refer to 17 and references therein.

The one remaining integral over the photon momentum can also be calculated. For that purpose, one has to take care of the energy-dependence of the width function in the $\mathrm{Z}$ boson propagator ( 13 ). Sufficiently far away from fermion thresholds, approximation ( 15) is excellent. This fact allows to apply the $\mathrm{Z}$ boson transformation thus ensuring that the $s$-dependence of the $\mathrm{Z}$ width does not complicate the analytic integrations:

$$
\begin{gathered}
\chi_{1}^{\prime}(s)=\chi_{1}(s), \\
\chi_{1}^{\prime}(s)=\frac{g_{1}^{2}}{4 \pi \alpha}\left(1+i \frac{\Gamma_{Z}}{M_{Z}}\right)^{-1} \frac{s}{s-M_{Z}^{\prime 2}+i M_{Z}^{\prime} \Gamma_{Z}^{\prime}}, \\
M_{Z}^{\prime}=M_{Z} / \sqrt{1+\frac{\Gamma_{Z}^{2}}{M_{Z}^{2}}} \simeq M_{Z}+\Delta_{Z}, \\
\Delta_{Z}=-\frac{1}{2} \frac{\Gamma_{Z}^{2}}{M_{Z}} \simeq-37 M e V, \\
\Gamma_{Z}^{\prime}=\Gamma_{Z} / \sqrt{1+\frac{\Gamma_{Z}^{2}}{M_{Z}^{2}}} \simeq \Gamma_{Z},
\end{gathered}
$$

where we used $\mathrm{M}_{Z}=91.1 \mathrm{GeV}$ and $\Gamma_{Z}=2.6 \mathrm{GeV}$. As a result, one gets for the total cross section ( 1 ) the QED correction functions $R_{T}^{e}(m, n)$ to order $\mathrm{O}(\alpha)$ and $\bar{R}_{T}^{e}(m, n)$ with soft photon exponentiation:

$$
\begin{gathered}
R_{T}^{e}(m, n)=1+S\left(\epsilon, \beta_{e}\right)+H_{2}^{e}(m, n)+H_{1}^{e}(m, n), \\
\bar{R}_{T}^{e}(m, n)=\left[1+\bar{S}\left(\beta_{e}\right)\right] H_{3}^{e}(m, n)+H_{1}^{e}(m, n) .
\end{gathered}
$$

The soft photon corrections $S\left(\epsilon, \beta_{e}\right)$ and $\bar{S}\left(\beta_{e}\right)$ have been introduced above. The hard photon parts consist of the residual hard part $H_{1}^{e}(m, n)$ and another one, $H_{2,3}^{e}(m, n)$, which depends on the treatment of the soft photon corner of the hard photon phase space integral:

$$
H_{i}^{e}(m, n)=g\left[K_{i}\left(R_{m}\right)-K_{i}\left(R_{n}^{*}\right)\right], \quad i=1,3 .
$$

Here, $g$ is a resonating kinematic factor:

$$
g=\frac{\left(1-R_{m}\right)\left(1-R_{n}^{*}\right)}{R_{m}-R_{n}^{*}}
$$

The threshold behaviour and further details of the dynamics are contained in the $K$-functions:

$$
\begin{gathered}
K_{1}(R)=-\frac{1}{2} \beta_{e} R[\Delta+(1+R) I(1-R)], \\
K_{2}(R)=+\frac{1}{1-R} \beta_{e}[R I(1-R)-I(0)], \\
K_{3}(R)=\frac{R}{1-R} \Delta^{\beta_{e}} J\left(\frac{\Delta}{1-R}\right), \\
I(z)=-\int_{\epsilon}^{\Delta} d v \frac{1}{v-z}=-\ln \frac{\Delta-z}{\epsilon-z},
\end{gathered}
$$




$$
J(\alpha)={ }_{2} F_{1}\left(1, \beta_{e}, 1+\beta_{e}, \alpha\right)=\beta_{e} \int_{0}^{1} d v \frac{v^{\beta_{e}-1}}{1-\alpha v} .
$$

The above definitions are valid if at least one of the $R_{m}, R_{n}$ are non-zero.

Although the limit of both masses $M_{m}, M_{n}$ vanishing formally exists, it differs from the correct form of $R_{T}^{e}(0,0)$ by a constant. This is due to the occurence of the double pole $(1-v)^{-2}$ from $\sigma_{T}^{e}\left(s^{\prime}, s^{\prime} ; 0,0\right)$ under the integral. Such a double pole behaviour cannot be calculated as continuous limit of the two single poles arising in the massive case:

$$
\bar{R}_{T}^{e}(0,0)=\left(\begin{array}{c}
\Delta^{\beta_{e}} \\
1
\end{array}\right)\left\{\begin{array}{c}
J(\Delta) \\
1
\end{array}\right\}\left[1+\bar{S}\left(\beta_{e}\right)+\delta_{2}^{V+S}\right]+\frac{2 \alpha}{\pi} Q_{e}^{2}\left(L_{e}-1\right)\left(\mp \frac{1}{2}\right) \ln \frac{\Delta}{1-\Delta},
$$

where the upper, barred (lower, unbarred) case is with (without) soft photon exponentiation. Further, if the cut-off $\Delta$ is removed, an additional finite error would occur due to lacking phase space factors ( 47). We quote here the corresponding correction without 20, 8 and with soft photon exponentiation:

$$
\begin{gathered}
\bar{R}_{T}^{e}(0,0)=\left\{\begin{array}{c}
J(\rho) \\
1
\end{array}\right\}\left[1+\bar{S}\left(\beta_{e}\right)+\delta_{2}^{V+S}\right]+\frac{2 \alpha}{\pi} Q_{e}^{2}\left(L_{e}-1\right)\left[\left(\mp \frac{1}{2}\right) \ln \frac{s}{m_{f}^{2}}+\frac{2}{3}\left(\begin{array}{c}
1 \\
-2
\end{array}\right)\right], \\
\rho=1-4 m_{f}^{2} / s .
\end{gathered}
$$

The logarithmic final state mass dependence regularises the massless photon propagator for emission of very hard photons and the corresponding singularity of $\ln (1-\Delta)$ in the limit $\Delta \rightarrow 1$. For applications, we add to the soft photon correction $\bar{S}\left(\beta_{e}\right)$ the next to leading order logarithmic correction $\delta_{2}^{V+S}$ exactly as obtained in $[15,16]$. For $H_{1}^{e}(1,1)$ which is the dominating hard photon contribution at LEP energies, we also add terms arising from an explicit integration with the corresponding hard photon terms in $\delta_{2}^{H}$ as quoted in 15, 16. This ensures the necessary high accuracy of the order $O(0.1 \%)$ but is not described here.

Due to the occurence of logarithms in the hard radiator function for $\sigma_{F B}$ (see 17] for details), the corresponding integrated expressions have a slightly more complicated structure than those for $\sigma_{T}$. For the photon exchange contribution,

$$
\begin{array}{r}
\bar{R}_{F B}^{e}(0,0)=\bar{R}_{T}^{e}(0,0)+\frac{2 \alpha}{\pi} Q_{e}^{2}\left\{\frac{1}{2}\left(L_{e}-1\right) \ln \frac{\Delta}{1-\Delta}\right. \\
\left.+4 d+\left(L_{e}-1\right)\left[L_{1}+L_{2}+2 d\right]+2 \frac{1-\Delta}{2-\Delta} L_{1}+4 d L_{2}+l_{4}+l_{3}\right\} .
\end{array}
$$

The following abbreviations are used:

$$
\begin{gathered}
d=\frac{1}{2} \frac{\Delta}{2-\Delta}, \\
L_{1}=\ln (1-\Delta), \\
L_{2}=\ln \left(1-\frac{\Delta}{2}\right), \\
l_{3}=L i_{2}(\Delta)-2 L i_{2}\left(\frac{\Delta}{2}\right), \\
l_{4}=L i_{2}\left(\frac{1}{2}\right)-L i_{2}\left(\frac{1}{2-\Delta}\right)-\ln 2 L_{2}+\frac{1}{2} L_{2}^{2},
\end{gathered}
$$




$$
\operatorname{Li}_{2}(z)=-\int_{0}^{1} \frac{d x}{x} \ln (1-x z) .
$$

The initial state correction to $\sigma_{F B}$ due to the $\gamma \mathrm{Z}$ interference is:

$$
\begin{array}{r}
\bar{R}_{F B}^{e}(1,0)=\left(\begin{array}{c}
\Delta^{\beta_{e}} \\
1
\end{array}\right)\left\{\begin{array}{c}
J[\Delta /(1-R)] \\
1
\end{array}\right\}\left[1+\bar{S}\left(\beta_{e}\right)+\delta_{2}^{V+S}\right] \\
+\frac{2 \alpha}{\pi} Q_{e}^{2}\left\{\left(\begin{array}{c}
0 \\
1
\end{array}\right)\left(L_{e}-1\right)\left[\ln \Delta+L_{R}(\Delta)\right]\right. \\
+2\left(L_{e}-1\right)\left[d r-\frac{1}{2} L_{R}(\Delta)\right]+4 d r+2 r \frac{1-\Delta}{2-\Delta} L_{1} \\
+\left[\frac{\left(L_{e}-1\right)}{1+R}(1+3 R)+4 d\right] r L_{2} \\
\left.+(1-2 R) l_{4}+l_{3}+2 R \frac{1+R^{2}}{(1+R)^{2}} D_{3}\right\} .
\end{array}
$$

The resonance logarithm $L_{R}(\Delta)$ as defined in (45) and the additional abbreviations depend on the complex $\mathrm{Z}$ boson mass parameter:

$$
\begin{gathered}
D_{3}=2 D_{2}-D_{1}+l_{4}+\left(L_{e}-1-2 \ln 2\right) L_{R}(\Delta), \\
D_{2}=L i_{2}\left(\frac{2}{1+R}\right)-L i_{2}\left(\frac{2-\Delta}{1+R}\right)+\ln 2 \ln (-r)-\left(L_{2}+\ln 2\right) \ln \left(1-\frac{2-\Delta}{1+R}\right), \\
D_{1}=L i_{2}\left(\frac{1}{R}\right)-L i_{2}\left(\frac{1-\Delta}{R}\right)-L_{1} \ln \left(1-\frac{1-\Delta}{R}\right), \\
r=\frac{1-R}{1+R} .
\end{gathered}
$$

The third correction to $\sigma_{F B}$ is due to $\mathrm{Z}$ exchange:

$$
\begin{gathered}
\bar{R}_{F B}^{e}(1,1)=\left(\begin{array}{c}
\Delta^{\beta_{e}} \\
1
\end{array}\right)\left\{\begin{array}{r}
\operatorname{Im}\left[R\left(1-R^{*}\right) J\left(\frac{\Delta}{1-R}\right)\right] / \operatorname{Im}(R) \\
1
\end{array}\right\}\left[1+\bar{S}\left(\beta_{e}\right)+\delta_{2}^{V+S}\right] \\
+\frac{2 \alpha}{\pi} Q_{e}^{2}\left\{\left(\begin{array}{c}
0 \\
1
\end{array}\right)\left(L_{e}-1\right)\left[\ln \Delta+t L_{R}(\Delta)\right]\right. \\
-t\left(L_{e}-1\right) L_{R}(\Delta)+2|r|^{2} d\left(L_{e}+1\right)+2 R \frac{1+R^{2}}{(1+R)^{2}} t D_{3}+2|r|^{2} \frac{1-\Delta}{2-\Delta} L_{1}+4 d|r|^{2} L_{2} \\
\left.+\left(L_{e}-1\right) L_{2}\left[8 \frac{R^{2}-1}{|1+R|^{4}}+4 \frac{1-6 R}{|1+R|^{2}}+5\right]+\left(-1+2|1-R|^{2}\right) l_{4}+l_{3}\right\}, \\
t=R \frac{2\left(1-R^{*}\right)}{R-R^{*}} .
\end{gathered}
$$

The C-odd initial state corrections behave very similar to those for $\sigma_{T}$. They have also the logarithmic electron mass singularity $L_{e}$. Both $\mathrm{Z}$ exchange corrections $R_{A}^{e}(1,1)$ develop a radiative tail beyond the resonace. This is due to their structure as already discussed for the angular distribution; see (45):

$$
R_{A}^{e}(1,1)=f_{A}^{1}(R)+f_{A}^{2}(R) t L_{R}(\Delta)\left(L_{e}-1\right) .
$$

Because of their complexity, we quote here the functions $R_{A}^{e}(1,0)$ and $R_{A}^{e}(1,1)$ for $\Delta=1$ taken at $R=1$, i.e. at resonance (we leave out here the coupling constants in the definition of $\chi(13)$ ):

$$
\left.|\chi|^{2} \operatorname{Re} R_{A}^{e}(1,1)\right|_{R=1} \sim \frac{1}{\rho^{2}}\left[1+\frac{\alpha}{\pi} Q_{e}^{2}\left(h_{1}^{e}+\rho h_{2}^{e}+\rho^{2} h_{A}^{e}\right)\right], \quad A=T, F B
$$




$$
\begin{gathered}
\rho=\Gamma_{Z} / M_{Z}, \\
h_{1}^{e}=-\frac{1}{2}+2 L i_{2}(1)+\left(L_{e}-1\right)\left[\frac{3}{2}+2 \ln \rho\right], \\
h_{2}^{e}=-2 \pi\left(L_{e}-1\right), \\
h_{T}^{e}=\left(L_{e}-1\right)(2-3 \ln \rho), \\
h_{F B}^{e}=\frac{9}{4}-\frac{7}{2} \ln 2+4 \ln ^{2} 2-\frac{3}{4} L i_{2}(1)-\frac{1}{2} \ln \rho+\left(L_{e}-1\right)\left(-\frac{3}{2} \ln 2+\frac{7}{2}-\frac{5}{2} \ln \rho\right) .
\end{gathered}
$$

From these $\mathrm{O}(\alpha)$ expressions it is easy to derive the corresponding ones for soft photon exponentiation. The most interesting feature is connected with hard photons - or better, with their absence at resonance. The initial state emission of a hard photon for $s=M_{Z}^{2}$ leads to a largely reduced effective energy $s^{\prime}$ and thus a non-resonant behaviour, i.e. a much reduced cross section. As a consequence of the resulting soft photon dominance, C-even and C-odd observables behave similar and consequently the leading order coefficients $h_{1}^{e}, h_{2}^{e}$ are equal for them at resonance. This has been observed numerically first in 42 and explained in 17. The $\gamma \mathrm{Z}$ interference corrections at the peak position are exclusively due to the imaginary parts of $R_{A}^{e}(1,0)$ since $\chi$ becomes imaginary for $R=1$ :

$$
\begin{gathered}
\operatorname{Re}\left[\chi R_{A}^{e}(1,0)\right] \sim \frac{1}{\rho}\left\{\delta_{A, T}+\frac{\alpha}{\pi} Q_{e}^{2}\left[\pi\left(L_{e}-1\right)+\rho g_{A}^{0}+\rho^{2} g_{A}^{1}\right]\right\}, \\
g_{T}^{0}=2\left(L_{e}-1\right)\left(\ln \rho-\frac{1}{2}\right), \quad g_{T}^{1}=-\frac{\pi}{2}\left(L_{e}-1\right), \\
g_{F B}^{0}=2\left(L_{e}-1\right)\left(\ln \rho-\frac{3}{2}+\ln 2\right)+\left[-2+2 \ln 2-4 \ln ^{2} 2+L i_{2}(1)\right], \\
g_{F B}^{1}=-\frac{\pi}{4} L_{e} .
\end{gathered}
$$

The Born contribution to $\sigma_{F B}$ vanishes at the peak. Nevertheless, the leading corrections to $\sigma_{T}$ and $\sigma_{F B}$ are equal again. Integrated cross section and asymmetry as functions of energy and the photon energy cut-off $\Delta$ have been studied in detail in 8.39 . We will come back to that point in chapters 5 and 6.

\section{INITIAL-FINAL STATE INTERFERENCE RADIATION}

The initial-final state interference corrections have an interesting property: only those which are diagonal in the arguments indicating the exchanged vector bosons are independent observables. The other interference contributions may be determined by the following simple relation:

$$
R_{A}^{i}(c ; m, n)=\frac{1}{2}\left[R_{A}^{i}(c ; m, m)+R_{A}^{i}(c ; n, n)^{*}\right]
$$

In order to get ( 91), it is essential to separate the reduced Born factor ( 10, 12) from the QED contents. The validity of ( 91) may be seen immediately from ( 3, 12, 13). An explicit proof has been given in 37.

\subsection{THE RADIATOR FUNCTIONS FOR THE ANGULAR DISTRIBUTIONS}


For interference corrections, the soft photon part has to include besides the soft photon emission terms $S_{A}(c, \epsilon, \lambda)$ also the contributions $B_{A}(c, \lambda ; m, n)$ originating from photon - $\mathrm{Z}$ boson and photon - photon box diagrams. Their sum is infrared finite (and independent of the infrared cut-off $\lambda$ ) while the infinitesimal soft photon cut-off parameter $\epsilon$ disappears only after integrating over the photon energy variable $v$ :

$$
\begin{gathered}
S_{A}^{i}(c, \epsilon ; m, n)=\frac{\alpha}{\pi} Q_{e} Q_{f}\left[S_{A}(c, \epsilon, \lambda)+B_{A}(c, \lambda ; m, n)\right], \\
S_{A}(c, \epsilon, \lambda)=2 D_{\bar{A}}(c)\left[2 \ln \frac{\epsilon}{\lambda} \ln \frac{c_{-}}{c_{+}}+L i_{2}\left(c_{+}\right)-L i_{2}\left(c_{-}\right)-\frac{1}{2}\left(\ln ^{2} c_{+}-\ln ^{2} c_{-}\right)\right] .
\end{gathered}
$$

Here, $D_{\bar{A}}(c)$ is defined in ( 32 - 33) with $\bar{A}=T(F B)$ if $A=F B(T)$, and $c_{ \pm}$in ( 41). The box diagram contributions 34 are dependent on the type of the gauge bosons exchanged. We write them in (anti-) symmetrised form:

$$
B_{T, F B}(c, \lambda ; m, n)=b(c, \lambda ; m, n) \pm b(-c, \lambda ; m, n) .
$$

The two different box functions are:

$$
\begin{gathered}
b(c, \lambda ; 0,0)=-2 c_{+}^{2} \ln \frac{c_{+}}{c_{-}}\left[4 \ln \frac{2 E}{\lambda}-2 \pi i\right]+\ln c_{-}\left[-2 c_{+}+c\left(\ln c_{-}+2 \pi i\right)\right]-2 \pi i c_{+}, \\
b(c, \lambda ; n, n)=-4 c_{+}\left(1-R_{n}\right)\left\{\ln \frac{c_{-}}{R_{n}}-\left(1-R_{n}\right) L_{n}+\right. \\
\left.\frac{1}{c_{+}}\left(1-R_{n}-2 c_{+}\right)\left[l(1)-l\left(c_{-}\right)-L_{n} \ln c_{-}\right]\right\} \\
-2 c_{+}^{2}\left\{\left[2 \ln \frac{2 E}{\lambda}+4 L_{n}+\ln \left(c_{+} c_{-}\right)\right] \ln \frac{c_{+}}{c^{-}}+2 l\left(c_{+}\right)-2 l\left(c_{-}\right)\right\}, \quad n \neq 0 .
\end{gathered}
$$

The following abbreviations are used:

$$
\begin{gathered}
l(a)=L i_{2}\left(1-a R_{n}^{-1}\right), \\
L_{n}=\ln \left(1-R_{n}^{-1}\right) \equiv L_{R_{n}}(1),
\end{gathered}
$$

and $R_{n}$ is defined in (46), $L_{R}(\Delta)$ in (45).

The hard radiator parts are independent of the gauge boson exchanged:

$$
H_{T, F B}^{i}(v, c)=\frac{\alpha}{\pi} Q_{e} Q_{f}\left[h_{T, F B}^{i}(v, c) \pm h_{T, F B}^{i}(v,-c)\right] .
$$

While the box terms for $A=F B$ and $A=T$ could be expressed by one and the same function, this is not the case here:

$$
\begin{aligned}
& h_{T}^{i}(v, c)= \\
& 2 c_{+}\left\{\left[\frac{4}{v}-3-z(2+z)\right] \ln \frac{c_{-}}{c_{+}}-(1+z)^{2} \ln \frac{c_{-}+z c_{+}}{c_{+}+z c_{-}}\right\} \\
& +2\left[-\frac{4}{v}+4+z(2+\ln z)\right]+\frac{4}{\gamma}\left[\frac{2}{v}-2-z(1+z)\right], \\
& h_{F B}^{i}(v, c)= \\
& +4 c_{+}\left[-\frac{4}{v}+4+2 z-z(1-z) \ln z\right]+\frac{4}{\gamma}\left(-\frac{2}{v^{2}}+\frac{5}{v}-3-z\right) \\
& +\frac{2}{\gamma^{2}}\left(-\frac{2}{v^{2}}+\frac{6}{v}-4-2 z-z^{2}\right)+2\left(z^{2}-1\right) c_{+} \ln c_{+} c_{-}+ \\
& 2\left[\left(1-z+z^{2}\right)+c\left(1-z^{2}\right)+c^{2}\left(1+z+z^{2}\right)\right] \ln \gamma .
\end{aligned}
$$


It is easy to check that in the soft photon limit $(v \rightarrow 0)$ the functions $h_{A}^{i}(v, c)$ behave such that they compensate after integration over the photon momentum the dependence of the box contributions on $\ln \epsilon$. In Fig. 3, the interference contributions are shown as functions of the scattering angle for three different energies. Compared to initial state radiation, the interference corrections are small. They do not contain fermion mass singularities though kinematic singularities at $\cos \Theta= \pm 1$ occur. In contrast to initial state radiation, these are integrable so that formally the full angular range in (22, 24) may be used. Of course, the interference contributions depend on the hard photon cut-off $\Delta$. For more severe cuts, they grow up. This is due to the fact that for the totally inclusive problem $\left(\Delta=v_{m} \sim 1\right)$ there exists a fine-tuned cancellation of box and bremsstrahlung contributions (for more details see next section) which becomes more and more disbalanced if the phase space of the bremsstrahlung integral becomes more and more restricted. For very tight cut values, $\Delta \ll 1$, the cross section to order $\mathrm{O}(\alpha)$ starts to diverge and may become even negative.

This can be seen from the leading soft photon contribution $R_{A}^{i, s o f t}(c ; m, n)$. After integration over the photon energy, the dependence of $S_{A}(c, \epsilon, \lambda)$ in $(92)$ on $\epsilon$ drops out. It is replaced by the following terms arising from the soft photon corner of the phase space integral:

$$
\begin{gathered}
R_{A}^{i, s o f t}(c ; m, n)=2 D_{\bar{A}}(c) \beta_{i} \ln \Delta, \\
\beta_{i}=2 \frac{\alpha}{\pi} Q_{e} Q_{f} \ln \frac{1-c}{1+c} .
\end{gathered}
$$

The complete interference contributions to the differential cross section may be found in the code MUCUTCOS [38. In analogy to initial state radiation, the logarithmic dependence on the cutoff $\Delta$ in ( 102) has to be cured by an adequate soft photon resummation which should lead to a replacement of

$$
\int_{0}^{\Delta} d v \sigma_{A}^{i, 0}\left(s, s^{\prime} ; m, n\right)\left[\delta(v) \beta_{i} \ln \epsilon+\Theta(v-\epsilon) \frac{\beta_{i}}{v}\right]
$$

by some function of the following type:

$$
\int_{0}^{\Delta} d v \sigma_{A}^{i, 0}\left(s, s^{\prime} ; m, n\right) \beta_{i} v^{\beta_{i}-1}
$$

In order to get really a smooth, well-defined behaviour of ( 105), the exponent therein has to be larger than -1 , i.e. $\beta_{i}$ should be positive definite. This is not the case in (105) as may be seen from (103). A possible way out is the exponentiation of interference radiation together with initial and final state radiation as is dictated by soft photon theorems (see, e.g., 10]). A collection of the relevant terms leads to the following order $\mathrm{O}(\alpha)$ expressions after integration. (One should have in mind that $s^{\prime}=s$ for the soft photon case):

$$
\frac{d \sigma^{\text {soft }}}{d c}=\sum_{A, m, n} \sigma_{A}^{0}(s, s ; m, n) D_{A}(c)\left\{1+\frac{2 \alpha}{\pi}\left[Q_{e}^{2} \ln \left(\frac{s}{m_{e}^{2}}-1\right)+2 Q_{e} Q_{f} \ln \frac{1-c}{1+c}+Q_{f}^{2}\left(\ln \frac{s}{m_{f}^{2}}-1\right)\right] \ln \Delta\right\} .
$$

The final state expressions have been taken from sect. 5. Again, the correction is not positive definite due to the expression in square brackets wich is a possible candidate to replace the exponent in (105). This is a consequence of too crude approximations. The soft photon contribution as a whole must be positive for any parameter combination since it is a complete module squared. In fact:

$$
\begin{gathered}
d \sigma^{\text {soft }}=\sum_{A, m, n} \sigma_{A}^{0}(s, s ; m, n) D_{A}(c)\left(1+\frac{\alpha}{\pi} \chi^{2}\right), \\
\chi=Q_{e}\left(\frac{k_{1}}{k_{1} p}-\frac{k_{2}}{k_{2} p}\right)+Q_{f}\left(\frac{p_{1}}{p_{1} p}-\frac{p_{2}}{p_{2} p}\right) .
\end{gathered}
$$


The four momenta used here are defined in ( 1). After a little algebra, one gets

$$
\begin{aligned}
& \chi^{2}= \\
& Q_{e}^{2}\left[\frac{s-2 m_{e}^{2}}{\left(-k_{1} p\right)\left(-k_{2} p\right)}-\frac{m_{e}^{2}}{\left(-k_{1} p\right)^{2}}-\frac{m_{e}^{2}}{\left(-k_{2} p\right)^{2}}\right]+Q_{f}^{2}\left[\frac{s-2 m_{f}^{2}}{\left(-p_{1} p\right)\left(-p_{2} p\right)}-\frac{m_{f}^{2}}{\left(-p_{1} p\right)^{2}}-\frac{m_{f}^{2}}{\left(-p_{2} p\right)^{2}}\right] \\
& +Q_{e} Q_{f}\left\{t_{+}\left[\frac{1}{\left(-k_{2} p\right)\left(-p_{1} p\right)}+\frac{1}{\left(-k_{1} p\right)\left(-p_{2} p\right)}\right]-t_{-}\left[\frac{1}{\left(-k_{1} p\right)\left(-p_{1} p\right)}+\frac{1}{\left(-k_{2} p\right)\left(-p_{2} p\right)}\right]\right\}, \\
& t_{+(-)}=-2 p_{1(2)} k_{2}=-2 p_{2(1)} k_{1}=\frac{s}{2} \pm \frac{c}{2} \sqrt{s-4 m_{e}^{2}} \sqrt{s-4 m_{f}^{2}} .
\end{aligned}
$$

After integration over the soft photon corner of the $\mathrm{n}$ dimensional photon momentum phase space 35, one gets for the corrections which are relevant for soft photon exponentiation:

$$
\begin{gathered}
d \sigma^{s o f t}=\sum_{A, m, n} \sigma_{A}^{0}(s, s ; m, n) D_{A}(c)\left\{1+\frac{2 \alpha}{\pi} Q_{e}^{2}\left(\frac{s-2 m_{e}^{2}}{\sigma_{e}} \ln \frac{s+\sigma_{e}}{s-\sigma_{e}}-1\right)\right. \\
+\frac{2 \alpha}{\pi} Q_{f}^{2}\left(\frac{s-2 m_{f}^{2}}{\sigma_{f}} \ln \frac{s+\sigma_{f}}{s-\sigma_{f}}-1\right) \\
\left.+\frac{2 \alpha}{\pi} Q_{e} Q_{f}\left[\frac{\sigma_{c}^{-}}{R^{-}} \ln \frac{\sigma_{c}^{-}+R^{-}}{\sigma_{c}^{-}-R^{-}}-\frac{\sigma_{c}^{+}}{R^{+}} \ln \frac{\sigma_{c}^{+}+R^{+}}{\sigma_{c}^{+}-R^{+}}\right]\right\} \\
\sigma_{e(f)}=\left(s^{2}-4 m_{e(f)}^{2} s\right)^{1 / 2} \\
\sigma_{c}^{ \pm}=s^{2} \pm c \sigma_{e} \sigma_{f} \\
R^{ \pm}=\left(\sigma_{c}^{ \pm 2}-16 m_{e}^{2} m_{f}^{2} s^{2}\right)^{1 / 2}
\end{gathered}
$$

In the ultra-relativistic limit of (111), one gets ( 106$)$. While ( 111) as a whole is positive definite, this property is lost in (106) as a result of the approximations.

Since we do not intend in our applications to apply such stringent cuts as to make inevitable the adequate exponentiation of initial final interference contributions we would like to remain at this point of clarification.

In a recent series of papers, there have been presented quite interesting results on soft photon exponentiation 43 including the interference [4]. Tracing back also to earlier work on that subject 10], the problem of exponentiation of soft photon interference radiation has been solved there in the ultra-relativistic limit. From the above discussion we conclude that this is adequate for values of $\cos \Theta$ not too close to 1, i.e. assuming some realistic acceptance cut for the experimental set-up. Further, the authors make the implicit assumption that one may use the radiators for $\sigma_{T}$ also for the differential cross section. This is not the case in general as shown in this article and in 17 . Nevertheless, the radiators for $\sigma_{T}$ can be used as an approximation. Further, we see no problem in combining the hard radiator parts presented here for the differential cross section with the result of 44 for the refined treatment of soft photon exponentiation.

\subsection{INTEGRATED CROSS SECTION AND FORWARD BACKWARD ASYMMETRY}

The integrated interference contributions are composed of box corrections $B_{A}(\lambda, n, n)$ and of bremsstrahlung contributions $b_{A}(\lambda, n, n)$ :

$$
R_{A}^{i}(n, n)=\frac{\alpha}{\pi} Q_{e} Q_{f}\left[B_{A}(\lambda ; n, n)+b_{A}(\lambda ; n, n)\right]
$$


For the total cross section one gets:

$$
\begin{gathered}
B_{T}(\lambda ; 0,0)=6 \ln \frac{2 E}{\lambda}-\frac{9}{2}, \\
b_{T}(\lambda ; 0,0)=-6 \ln \frac{2 E \Delta}{\lambda}+3(2+\Delta), \\
B_{T}(\lambda ; 1,1)=6 \ln \frac{2 E}{\lambda}-9+3 R\left[1+(1+R) L_{R}(1)\right]+3 L_{Z}, \\
b_{T}(\lambda ; 1,1)=-6 \ln \frac{2 E \Delta}{\lambda}+6+3\left[\Delta(1-R)-R(1+R) L_{R}(\Delta)\right], \\
L_{Z}=\ln \left(s / M_{Z}^{\prime 2}\right),
\end{gathered}
$$

where $L_{R}(\Delta)$ is defined in (45) and $M^{\prime}$ in ( 53). Without cut $(\Delta=1)$, the resulting corrections to $\sigma_{T}$ are quite small 8 , 41 :

$$
\begin{gathered}
R_{T}^{i}(0,0)=\frac{\alpha}{\pi} Q_{e} Q_{f} \frac{9}{2}, \\
R_{T}^{i}(1,1)=\frac{\alpha}{\pi} Q_{e} Q_{f} 3 L_{Z} .
\end{gathered}
$$

The interference QED corrections due to photon exchange to the antisymmetric cross section part $\sigma_{F B}$ are:

$$
\begin{array}{r}
B_{F B}^{i}(\lambda ; 0,0)=(1+8 \ln 2) \ln \frac{2 E}{\lambda}-\frac{3}{4}\left(1+6 \ln 2+\ln ^{2} 2\right)+\frac{1}{2} i \pi(2-5 \ln 2), \\
b_{F B}^{i}(\lambda ; 0,0)=-(1+8 \ln 2) \ln \frac{2 E \Delta}{\lambda}+\frac{1}{4}(2-5 \Delta)+\frac{1}{4}\left(4+26 \Delta-5 \Delta^{2}\right) \ln 2 \\
\quad+\frac{1}{4}\left(-44+26 \Delta-5 \Delta^{2}\right) L_{2}+\frac{1}{4}\left(15-20 \Delta+5 \Delta^{2}\right) L_{1} \\
+\frac{3}{4} L i_{2}(1)+4 \ln ^{2} 2-4 L i_{2}(\Delta)+8 L i_{2}\left(\frac{\Delta}{2}\right)-\frac{5}{2} L i_{2}(\Delta-1) .
\end{array}
$$

Their sum is a simple constant for $\Delta=123$ :

$$
R_{F B}^{i}(0,0)=\frac{\alpha}{\pi} Q_{e} Q_{f}(4.572-i 2.302) .
$$

The $\mathrm{Z}$ exchange corrections are much more involved. For $\Delta=1$, they may be found in 8 ; with $\Delta \leq 1$ :

$$
\begin{array}{r}
B_{F B}^{i}(\lambda ; 1,1)=(1+8 \ln 2) \ln \frac{2 E}{\lambda}-\frac{3}{2}+R-\left(9-4 R-4 R^{2}\right) \ln 2 \\
-2 \ln ^{2} 2+\frac{1}{2}(5-4 R) L_{Z}-\frac{1}{2}\left[4-9 R+3 R^{2}+2\left(-5+3 R-6 R^{2}\right) \ln 2\right] L_{R}(1) \\
-\left(1-3 R+6 R^{2}-8 R^{3}\right)\left[L i_{2}\left(1-\frac{1}{2 R}\right)-L i_{2}\left(1-\frac{1}{R}\right)\right]-4 R^{3}\left[L i_{2}(1)-L i_{2}\left(1-\frac{1}{R}\right)\right], \\
b_{F B}^{i}(\lambda ; 1,1)=-(1+8 \ln 2) \ln \frac{2 E \Delta}{\lambda}+\frac{1}{4}(2-5 \Delta+5 R \Delta) \\
+\frac{1}{4}\left(4-16 R \Delta+5 R \Delta^{2}-10 R^{2} \Delta+26 \Delta-5 \Delta^{2}\right) \ln 2+4 \ln ^{2} 2+2 L i_{2}(1)
\end{array}
$$




$$
\begin{array}{r}
+\frac{5}{4}\left(3-R+2 R \Delta-R \Delta^{2}-2 R^{2}+2 R^{2} \Delta-4 \Delta+\Delta^{2}\right) L_{1} \\
+\left(-5+3 R-4 R \Delta+\frac{5}{4} R \Delta^{2}+5 R^{2}-\frac{5}{2} R^{2} \Delta-\frac{6}{1+R}+\frac{13}{2} \Delta-\frac{5}{4} \Delta^{2}\right) L_{2} \\
+R\left(-5+\frac{6}{1+R}+R\right) L_{R}(\Delta)-4 L i_{2}(\Delta)+8 L i_{2}\left(\frac{\Delta}{2}\right) \\
+\frac{R}{2}\left(3+5 R^{2}\right) D_{1}-\frac{1}{2}\left(5+3 R+3 R^{2}+5 R^{3}\right) D_{2}
\end{array}
$$

with $D_{1,2}$ defined in (75 76). Again, the $\mathrm{Z}$ boson parameters have to be understood in primed quantities. A generalization of the initial final interference QED corrections to the case of several massive gauge bosons 12] is trivial due to relation (91). At the $\mathrm{Z}$ peak, a fine-tuned cancellation of box and bremsstrahlung terms occurs. Therefore, the resulting $\mathrm{Z}$ exchange interference contributions $R_{T}^{i}(1,1)$ and $R_{F B}^{i}(1,1)$ become small there. This has been noticed from numerical results in 7. 8, 42 and is known also from earlier investigations of $\mathrm{J} / \psi$ physics 45 . For $R_{T}^{i}(1,1)$ it is evident from ( 122). A Taylor expansion around $s=M_{Z}^{2}$ yields:

$$
\begin{gathered}
R_{T}^{i}(1,1)=\frac{\alpha}{\pi} Q_{e} Q_{f}\left[6 \ln \frac{R-1+\Delta}{\Delta}+9(R-1) \ln (R-1+\Delta)+O(R-1)\right], \\
R_{F B}^{i}(1,1)=\frac{\alpha}{\pi} Q_{e} Q_{f}\left\{[1+8 \ln (2-\Delta)-4 \ln (1-\Delta)] \ln \frac{R-1+\Delta}{\Delta}-3(\ln 2)(R-1) \ln (R-1)\right. \\
\left.+\frac{3}{2}[1+8 \ln (2-\Delta)-6 \ln (1-\Delta)](R-1) \ln (R-1+\Delta)+4(R-1) \ln (1-\Delta)+O(R-1)\right\} .
\end{gathered}
$$

For $\Delta$ approaching 1 and using in the peak region $\ln R=R-1+O\left[(R-1)^{2}\right]$, one gets

$$
\begin{gathered}
R_{T}^{i}(1,1 ; \Delta=1)=\frac{\alpha}{\pi} Q_{e} Q_{f}\left\{-3(R-1)+O\left[(R-1)^{2}\right]\right\}, \\
R_{F B}^{i}(1,1 ; \Delta=1)=\frac{\alpha}{\pi} Q_{e} Q_{f}\{-3(R-1)(\ln 2) \ln (R-1) \\
\left.+\frac{1}{4}\left[7-10 \ln 2-6 \ln ^{2} 2-6 L i_{2}(1)\right](R-1)+O\left[(R-1)^{2}\right]\right\} .
\end{gathered}
$$

The real parts of $R_{A}^{i}(1,1)$ contribute to $\sigma_{A}$ in a product together with $|\chi|^{2} \sim|R-1|^{-2}$. As a cosequence of their proportionality to $(R-1)$ whose real part vanishes at the peak, the $\mathrm{Z}$ exchange interference corrections become extremely small. For $\Delta \neq 1$, there are at the peak larger contributions due to $\ln (R-1+\Delta) \sim \ln \Delta$ in $(128$, 129).

A similar discussion applies to the $\gamma \mathrm{Z}$ interference corrections. Due to ( 91), half the $R_{A}^{i}(1,1)$ are combined there with the resonating function $\chi$ wich becomes imaginary at $R=1$. So, the imaginary parts of $R_{A}^{i}(1,1)$ are relevant. Again, the QED corrections are suppressed compared to the $\mathrm{Z}$ exchange Born cross section behaving like $|1-R|^{-2}$.

So, all the interference contributions are small since the photonic corrections $R_{A}^{i}(0,0)$ are also non-resonanting at the peak. In case of cuts to the photon energy, $\Delta<1$, the $\mathrm{O}(\alpha)$ interference bremsstrahlung has to be taken into account properly. For very small $\Delta$, logarithms of the type $\ln \Delta$ may become even dominating and then one should try to apply some soft photon summation procedure. 


\section{FINAL STATE RADIATION}

\subsection{THE RADIATOR FUNCTIONS FOR THE ANGULAR DISTRIBUTION}

The final state radiation contributions to the differential cross section have a simple angular dependence compared to the expressions discussed in the preceding sections. The soft photon parts of the final state radiators $S_{A}^{f}(c, \epsilon ; m, n)$ may be obtained from $S\left(\epsilon, \beta_{e}\right)$ as defined in ( 34) by replacing $m_{e}$ by the final state mass $m_{f}$ :

$$
S_{A}^{f}(c, \epsilon ; m, n)=D_{A}(c) S\left(\epsilon, \beta_{f}\right), \quad A=T, F B,
$$

where $D_{A}(\mathrm{c})$ is introduced in $(32,33)$. The hard photon radiators are:

$$
\begin{gathered}
H_{T}^{f}(v, c)=\frac{\alpha}{\pi} Q_{f}^{2}\left\{D_{T}(c)\left[H_{f}(v)-3 v\right]+4 v\right\}, \\
H_{F B}^{f}(v, c)=\frac{\alpha}{\pi} Q_{f}^{2} D_{F B}(c) h_{f}(v),
\end{gathered}
$$

where the radiators $H_{f}(v), h_{f}(v)$ have been derived earlier for the integrated cross sections $\sigma_{T}$ and $\sigma_{F B}$ 17]:

$$
\begin{gathered}
H_{f}(v)=\frac{1+(1-v)^{2}}{v}\left[L_{f}-1+\ln (1-v)\right], \\
h_{f}(v)=\frac{2}{v}\left[(1-v)\left(L_{f}-1\right)+\ln (1-v)+\frac{1}{2} v^{2} L_{f}\right] .
\end{gathered}
$$

The integration of the symmetric radiator function $(A=T)$ over the photon energy gives a contribution to the differential cross section:

$$
R_{T}^{f}(c ; m, n)=\frac{1}{4}\left(5-3 c^{2}\right) .
$$

The corresponding anti-symmetric radiator integral vanishes:

$$
R_{F B}^{f}(c ; m, n)=0 .
$$

As has been pointed out e.g. in 19, the soft photon corrections ( 132 ) to even and odd cross section parts are equal and will, consequently, cancel for the forward backward asymmetry. After integration over the full photon phase space, eqs. (137- 138), there is minor influence on $A_{F B}$ due to the correction in the denominator. Nevertheless, for small cut off values $\Delta \ll 1$, soft photon exponentation is recommended in order to get reliable numerical results. A naive addition of initial and final state radiation corrections as has been performed in 17] changes this dependence and leads to an overestimation of final state radiation due to an inbalance of corrections. In order to maintain the smallness of isolated final state corrections after combination with the large contributions from the initial state, one has to proceed more carefully. A treatment based on the intuitive picture of subsequent radiation of photons from initial and final states is proposed in subsection 5.3. An isolated soft photon exponentiation for final state radiation is analogue to the procedure described in 15. and consists of the replacements

$$
\delta(v) S_{A}^{f}(c, \epsilon ; m, n) \rightarrow \beta_{f} v^{\beta_{f}-1} D_{A}(c) \bar{S}\left(\beta_{f}\right),
$$




$$
H_{A}^{f}(v, c) \rightarrow \bar{H}_{A}^{f}(v, c)=H_{A}^{f}(v, c)-\frac{\beta_{f}}{v} D_{A}(c) .
$$

Numerical results due to final state radiation are shown in fig.4.

5.2. INTEGRATED CROSS SECTION AND FORWARD BACKWARD ASYMMETRY TO ORDER $\mathrm{O}(\alpha)$

The integrated final state $\mathrm{O}(\alpha)$ QED corrections are simple:

$$
\begin{gathered}
R_{A}^{f}(m, n)=\frac{\alpha}{\pi} Q_{f}^{2}\left[\frac{1}{2}\left(L_{f}-1\right)\left(4 \ln \Delta+3-4 \Delta+\Delta^{2}\right)-2 L i_{2}(\Delta)+2 L i_{2}(1)+r_{A}^{f}(\Delta)\right], \\
r_{T}^{f}(\Delta)=\frac{1}{2}(1-\Delta)(3-\Delta) L_{1}+\frac{1}{4}\left(6 \Delta-2-\Delta^{2}\right), \\
r_{F B}^{f}(\Delta)=\frac{1}{2}\left(\Delta^{2}-1\right) .
\end{gathered}
$$

If the photon energy cut is removed, one gets:

$$
\begin{gathered}
R_{T}^{f}(m, n)=\frac{\alpha}{\pi} Q_{f}^{2} \frac{3}{4}, \\
R_{F B}^{f}(m, n)=0 .
\end{gathered}
$$

Figs. 5 and 6 illustrate the above discussion of the corrections to the angular distribution due to final state radiation. A comparison of (138) with ( 136, 140) shows that the contribution of final state radiation to $\sigma_{F B}$ vanishes if neither a cut on the photon energy is applied nor finite mass effects are taken into account 46. Then, the only (and minor) influence of final state radiation on $A_{F B}$ is due to the C-even correction in ( [) . If a tight cut is applied $(\Delta \ll 1)$, the C-even and C-odd final state corrections ( 141. 143) approach each other because from soft photons there is no influence on the angular behaviour of the emitted fermions. Due to the logarithmic cut dependence, one again should exponentiate the soft photon contributions in that case but we leave out the details here. The ansatz is defined in (139-140).

\subsection{SOFT PHOTON EXPONENTATION}

As may be seen from ( 133 - 136), the final state radiation contributions to the differential cross section behave as a function of the normalised photon energy $v$ quite similar to the integrated

quantities $\sigma_{A}, A=T, F B$. Based on their simple dependence on the scattering angle, we now derive a common treatment of soft photon exponentiation for initial and final state corrections. We start from the definition ( 2, 3):

$$
\begin{array}{r}
\frac{d \sigma^{(e+f)}}{d c}=\sum_{m, n=0,1} \sum_{A=T, F B} R e\left\{\int_{0}^{\Delta} d v \sigma_{A}^{0}\left(s^{\prime}, s^{\prime} ; m, n\right)\right. \\
\left.\left\{\delta(v)\left[1+S\left(\epsilon, \beta_{e}\right)\right) D_{A}(c)\right]+\theta(v-\epsilon) H_{A}^{e}(v, c)\right\} \\
\left.+\sigma_{A}^{0}(s, s ; m, n) D_{A}(c) \int_{0}^{\Delta} d u\left[\delta(u) S\left(\epsilon, \beta_{f}\right)+\theta(u-\epsilon) \tilde{H}_{A}^{f}(u, c)\right]\right\},
\end{array}
$$




$$
H_{A}^{f}(u, c)=D_{A}(c) \tilde{H}_{A}^{f}(u, c),
$$

where $\tilde{H}_{F B}^{f}$ is independent of the scattering angle and $\tilde{H}_{T}^{f}$ has an almost negligible dependence. Further, (146) can be rewritten as follows:

$$
\begin{array}{r}
\frac{d \sigma^{(e+f)}}{d c}=\sum_{m, n=0,1} \sum_{A=T, F B} \operatorname{Re} \int_{0}^{\Delta} d v \sigma_{A}^{0}\left(s^{\prime}, s^{\prime} ; m, n\right) \\
\left\{\delta(v)\left[1+S\left(\epsilon, \beta_{e}\right)+\tilde{r}_{A}^{f}\left(s^{\prime}, c, \Delta\right)\right] D_{A}(c)+\theta(v-\epsilon) H_{A}^{e}(v, c)\right\}, \\
\tilde{r}_{A}^{f}(s, c, \Delta)=\int_{0}^{\Delta} d u\left[\delta(u) S\left(\epsilon, \beta_{f}\right)+\theta(u-\epsilon) \tilde{H}_{A}^{f}(u, c)\right] .
\end{array}
$$

The dependence of $\tilde{r}_{A}^{f}$ on $c$ is very weak for $A=T$ and absent for $A=F B$. Now the following ansatz seems to include a quite reasonable approximation of higher order soft photon corrections:

$$
\begin{gathered}
\frac{d \bar{\sigma}^{(e+f)}}{d c}=\sum_{m, n=0,1} \sum_{A=T, F B} \operatorname{Re} \int_{0}^{\Delta} d v \sigma_{A}^{0}\left(s^{\prime}, s^{\prime} ; m, n\right) F_{A}^{e}(v, c) F_{A}^{f}\left(v, c, s^{\prime}\right), \\
F_{A}^{e}(v, c)=\delta(v) D_{A}(c)\left[1+S\left(\epsilon, \beta_{e}\right)\right]+\theta(v-\epsilon) H_{A}^{e}(v, c) \\
F_{A}^{f}\left(v, c, s^{\prime}\right)=1+\tilde{r}_{A}^{f}\left(s^{\prime}, c, \Delta\right) .
\end{gathered}
$$

The exponentiation of soft photon contributions leads to the following replacements:

$$
\begin{gathered}
F_{A}^{e}(v, c) \rightarrow \bar{F}_{A}^{e}(v, c)=D_{A}(c) \beta_{e} v^{\beta_{e}-1}\left[1+\bar{S}\left(\beta_{e}\right)\right]+\bar{H}_{A}^{e}(v, c), \\
F_{A}^{f}\left(v, c, s^{\prime}\right) \rightarrow \bar{F}_{A}^{f}\left(v, c, s^{\prime}\right)=\int_{0}^{\Delta^{\prime}} d u\left\{\beta_{f}^{\prime} u^{\beta_{f}^{\prime}-1}\left[1+\bar{S}\left(\beta_{f}^{\prime}\right)\right]+\overline{\tilde{H}}_{A}^{f}\left(u, c, \beta_{f}^{\prime}\right)\right\} \\
\beta_{f}^{\prime}=\frac{2 \alpha}{\pi} Q_{f}^{2}\left(\ln \frac{s^{\prime}}{m_{f}^{2}}-1\right) .
\end{gathered}
$$

Here again the residual hard radiator parts $\bar{H}$ are

$$
\overline{\tilde{H}}_{A}^{a}(v, c, \beta)=\tilde{H}_{A}^{a}(v, c, \beta)-\frac{\beta}{v} D_{A}(c) .
$$

The integrations over the variable $u$ can be performed in ( 154):

$$
\begin{gathered}
\bar{F}_{A}^{f}\left(v, c, s^{\prime}\right)=\Delta^{\prime \beta_{f}^{\prime}}\left[1+\bar{S}\left(\beta_{f}^{\prime}\right)\right]+G_{A}\left(v, c, s^{\prime}\right), \\
G_{A}\left(v, c, s^{\prime}\right)=\frac{1}{4} \beta_{f}^{\prime} \Delta^{\prime}\left(\Delta^{\prime}-4\right)-\frac{\alpha}{\pi} Q_{f}^{2}\left[2 L i_{2}\left(\Delta^{\prime}\right)+g_{A}\left(\Delta^{\prime}, c\right)\right], \\
g_{T}\left(\Delta^{\prime}, c\right)=\frac{1}{2}\left(1-\Delta^{\prime}\right)\left(3-\Delta^{\prime}\right) \ln \left(1-\Delta^{\prime}\right)-\frac{1}{4} \Delta^{\prime}\left(\Delta^{\prime}-6\right)-\left(3-\frac{4}{1+c^{2}}\right) \frac{\Delta^{\prime 2}}{2} . \\
g_{F B}\left(\Delta^{\prime}, c\right)=\frac{1}{2} \Delta^{\prime 2},
\end{gathered}
$$

In the above formulae, we introduced the variable $\Delta^{\prime}$ which is connected with the reduced $s^{\prime}$ in the same way as $\Delta$ with $s$ :

$$
\begin{gathered}
\Delta^{\prime} \equiv 1-\frac{s_{\min }^{\prime}}{s^{\prime}}, \\
\Delta^{\prime}=\frac{\Delta-v}{1-v} .
\end{gathered}
$$


if one wants to obtain from the above expressions for the angular distribution those for $\sigma_{T}$ and $A_{F B}$, it is helpful to start with the observation that the $\bar{F}_{A}^{f}$ are independent of the scattering angle with exclusion of $g_{T}\left(\Delta^{\prime}, c\right)$. Since that dependence is minor, i.e. less than $\frac{1}{2} \frac{\alpha}{\pi} Q_{f}^{2}=0.1 \% Q_{f}^{2}$, one can neglect it. Then the final state radiation factor is completely independent of the scattering angle and the integration over c has to be done for $\mathrm{F}_{A}^{e}(\mathrm{v}, \mathrm{c})$ only:

$$
\begin{gathered}
\bar{\sigma}_{A}^{(e+f)}=\sum_{m, n=0,1} R e \int_{0}^{\Delta} d v \sigma_{A}^{0}\left(s^{\prime}, s^{\prime} ; m, n\right) \bar{F}_{A}^{e}(v) \bar{F}_{A}^{f}\left(v, s^{\prime}\right), \\
\bar{F}_{A}^{e}(v)=\frac{3}{4} \int_{0}^{1} d c F_{A}^{e}(v, c) .
\end{gathered}
$$

This integration yields the normal convolution representation for the total cross section $\bar{\sigma}_{A}$, but now modified by the additional factor $\bar{F}_{A}^{f}$. So, by a direct Feynman diagram calculation we have shown that (163) is an approximation of sufficient accuracy though some well-defined terms of the order $\mathrm{O}(\alpha)$ have been neglected. A similar formula in the case of $\sigma_{T}$ has been obtained by other methods in 14 . The dependence of $\sigma_{T}$ and $A_{F B}$ on the cut-off parameter $\Delta$ is shown in figs. 5 and 6 for different handlings of initial and final state corrections. For smaller $\Delta$ the cross section becomes smaller since the positive hard photon part is restricted then. In the tail region (figs. $5 \mathrm{c}, 6 \mathrm{c}$ ), one observes a steeply falling cross section at values of $\Delta$ which cut away the radiative tail. This happens if the effective energy $s^{\prime}$ cannot reach the resonance energy, i.e. $\Delta \leq 1-\mathrm{M}_{Z}^{2} / \mathrm{s}$. Even for infinitesimal $\Delta$ the net negative bremsstrahlung corrections remain finite due to soft photon exponentiation as discussed in the text. In the figures, we applied the simplified but sufficiently accurate procedure explained in chapter 4.2. Since the corresponding soft photon corrections in units of the Born cross sections $\sigma_{T}$ and $\sigma_{F B}$ are equal, the net final state correction to $A_{F B}$ vanishes for vanishing $\Delta$. For sufficiently large cut-off values, the final state contributions also remain nearly negligible. In the intermediate region, they must be taken into account at least for precision measurements.

\section{DISCUSSION}

In the foregoing chapters, basic formulae for and characteristic features of the QED corrections to three different observables in fermion pair production from $\mathrm{e}^{+} \mathrm{e}^{-}$annihilation have been presented separately. Numerical results have been produced with the codes MUCUT and MUCUTCOS of the package ZBIZON 38 .

In fig. 7, the net QED corrections to the differential cross section are shown in combination with the weak loop corrections. The latter have been determined with the code DIZET which is also part of ZBIZON. For $M_{Z}=91.1, m_{t}=100 ., M_{H}=100$. (all masses in $\mathrm{GeV}$ ), and $\alpha_{s}=0.12$ we get in accordance with 27] [16]: $\sin ^{2} \Theta_{W}=0.2314, \Gamma_{Z}=2.477 \mathrm{GeV}$. The corrected muon decay constant, running QED coupling, and weak neutral vector and axial vector couplings to be used in ( 16, 17, 20, 21) are 29.

$$
\begin{gathered}
\alpha F_{A}\left(M_{Z}^{2}\right)=\alpha(1.063-i 0.018), \\
G_{\mu} \rho_{Z}\left(M_{Z}^{2}\right)=G_{\mu}(1.000-i 0.005), \\
a_{e}=-\frac{1}{2} \\
v_{e}\left(M_{Z}^{2}\right)=-\frac{1}{2}\left[1-4 \sin ^{2} \Theta_{W}(1.011+i 0.013)\right],
\end{gathered}
$$




$$
v_{e e}\left(M_{Z}^{2}\right)=\frac{1}{4}\left[1-8 \sin ^{2} \Theta_{W}(1.011+i 0.013)+16 \sin ^{4} \Theta_{W}(1.023+i 0.027)\right] .
$$

If, for the sake of comparison, the initial-final interference terms are excluded, the fully corrected total cross section $\sigma_{T}$ as obtained here agrees within $\pm 0.2 \%$ with that obtained from the code ZSHAPE 15 which is exact to order $\mathrm{O}\left(\alpha^{2}\right)$. For $A_{F B}$ and $\mathrm{d} \sigma / \mathrm{d} \cos \Theta$, we do not know of a program which would allow for a similar comparison.

While the weak loop corrections remain small 16], the QED corrections amount typically to several percents or more, essentially due to initial state radiation. Even in the tail region, the angular dependence of the corrected differential cross section follows closely the Born cross section behaviour with exclusion of scattering angles near $|\cos \Theta|=1$. This is reflected also in figs. 8 and 9 where $\sigma_{T}$ and $A_{F B}$ are shown as functions of an acceptance cut, $|\Theta|<\Theta_{\max }$. In general, the asymmetry is not a monotonic, rising with $\Theta_{\max }$ function though there are regions with an almost linear behaviour. For loose cut values, the tail effect is pronounced at $s>M_{Z}^{2}$. The total asymmetry is small at resonance with corrections nearly of the order of the Born contribution. The relatively large asymmetry value for a tight cut, $E_{\gamma}<1 \mathrm{GeV}$, may be understood from figs. $2 \mathrm{~b}$ where a systematic distortion of the differential cross section is present in that case.

In some recent determinations of $\sigma_{T}$ 近 and $A_{F B}$ 25] at LEP, the QED corrections 11] 158 have been applied to data corrected to correspond to a detector with a full angular acceptance $(4 \pi$ geometry). From figs. 8,9 one can see that this may be justified although the present formulae allow a refined analytic approach to the data. This seems to be recommended at least for the interpretation of precision measurements.

In view of the relative smallness of interference and final state corrections, it is interesting to know where they can be neglected completely. Of course, this depends on the energy region and photon energy cut-off chosen. In figs. 10,11 corresponding regions of relevance are shown.

To summarise, the analytic approach to QED corrections has been proven quite powerful both for consistency checks of Monte Carlo programs $[3$ and for the interpretation of experimental data. This article contains a first, systematic presentation of our analytic formulae for the complete set of QED corrections to the differential cross section $\mathrm{d} \sigma / \mathrm{d} \cos \Theta$, total cross section $\sigma_{T}$ and integrated forward backward asymmetry $A_{F B}$.

\section{ACKNOWLEDGMENTS}

We would like to thank F.A. Berends, M. Greco, W.Hollik, R.Kleiss and Yu. Sedykh for enligthening discussions and fruitful cooperation. 


\section{FIGURE CAPTIONS}

Figure caption Fig. 1. The C-even and C-odd contributions to $\mathrm{d} \sigma / \mathrm{d} \cos \Theta$ due to $\mathrm{Z}$ exchange (solid line), $\gamma$ exchange (dashed line), $\gamma \mathrm{Z}$ interference (dotted line) in nbarn as functions of the scattering angle at $\sqrt{s}=30 \mathrm{GeV}$ (a) $91.1 \mathrm{GeV}$ (b), $200 \mathrm{GeV}$ (c). Parameters: $M_{Z}=91.1 \mathrm{GeV}, \Gamma_{Z}$ $=2.5 \mathrm{GeV}, \sin ^{2} \Theta_{W}=.23, \Delta=1$.

Fig. 2. The differential cross section with Born and initial state radiation contributions in nbarn as functions of the scattering angle and of the photon energy cut-off $\Delta$. Other parameters: see fig.1.

Fig.3. QED contribution to $\mathrm{d} \sigma / \mathrm{d} \cos \Theta$ due to initial-final interference as function of the scattering angle and $\Delta$. Parameters: see figs.1,2.

Fig. 4. Born plus final state correction contributions to $\mathrm{d} \sigma / \mathrm{d} \cos \Theta$ in nbarn as function of the scattering angle and $\Delta$. Parameters: see figs.1,2.

Fig. 5. The total cross section $\sigma_{T}$ as function of the photon energy cut-off $\Delta$. Parameters: see figs.1,2.

Fig. 6. The integrated forward-backward asymmetry $A_{F B}$ as function of the photon energy cut-off $\Delta$. Parameters: see figs.1,2.

Fig. 7. The differential cross section with complete electroweak corrections as function of the scattering angle and $\Delta$. Parameters $M_{Z}=91.1, m_{t}=100 ., M_{H}=100$. (all masses in GeV), $\alpha_{s}=0.12$.

Fig. 8.The total cross section $\sigma_{T}$ as function of an acceptance cut on the scattering angle, $|\cos \Theta| \leq \cos \Theta_{\max }$. Parameters see fig. 7 .

Fig. 9. The integrated forward-backward asymmetry $A_{F B}$ as function of an acceptance cut on the scattering angle. Parameters see fig. 7.

Fig. 10. Cross section contributions due to initial-final state interference (a) and final state radiation (b) in percent as functions of $\sqrt{s}$ and $\Delta$. Parameters: see figs.1,2.

Fig. 11. Asymmetry contributions due to initial-final state interference (a) and final state radiation (b). Parameters: see fig. 10 . 


\section{References}

[1] S.L. Glashow, Nucl. Phys. 22(1961)579;

S. Weinberg, Phys. Rev. Letters 19(1967)1264;

A. Salam, in Elementary Particle Theory, ed. N. Svartholm (Stockholm 1968) p. 367.

[2] G. Altarelli, R. Kleiss, C. Verzegnassi (eds.), Z Physics at LEP 1, CERN report CERN 89-08 (Sept. 1989).

[3] R. Kleiss et al., Monte Carlos for Electroweak Physics, in [2] vol.3, p.1.

[4] G.S. Abrams et al., MARK II Collab., Phys. Rev. Lett. 63(1989)724;

B. Adeva et al., L3 Collab., Phys. Letters B231(1989)509;

D. Decamp et al., ALEPH Collab.,Phys. Letters B231(1989)519;

M. Akrawy et al., OPAL Collab.,Phys. Letters B231(1989)530;

P. Aarnio et al., DELPHI Collab.,Phys. Letters B231(1989)539;

[5] G. Bonneau, F. Martin, Nucl. Phys. B27(1971)381.

[6] F.A. Berends, R. Kleiss, Nucl. Phys. B177(1981)237; B260(1985)32.

[7] F.A. Berends, R. Kleiss, S. Jadach, Nucl. Phys. B202(1982)63; Comput. Phys. Commun. $29(1983) 185$.

[8] D. Bardin, M. Bilenky, O. Fedorenko, T. Riemann, JINR Dubna prepr. E2-87-663(1987), E288-324(1988).

[9] D. Bardin, A. Leike, T. Riemann, M. Sachwitz, Phys. Letters B 206(1988)539.

[10] M. Greco, G. Pancherivi, Y. Srivastava, Nucl. Phys. B101(1975)11; ibid, B171(1980)118; E: Nucl. Phys. B197(1982)543.

[11] R.N. Cahn, Phys. Rev. D36(1987)2666;

A. Borrelli, M. Consoli, L. Maiani, R. Sisto, Nucl. Phys. B333(1990)357.

[12] A. Leike, T. Riemann, M. Sachwitz, Phys. Letters B241(1990)267.

[13] E. Kuraev, V. Fadin, Sov. J. Nucl. Phys. 41(1985)466;

G. Altarelli, G. Martinelli, in Physics at LEP, eds. J. Ellis et al., CERN report CERN 8602(1986), vol 1. p. 47.

[14] O. Nicrosini, L. Trentadue, Phys. Letters 196B(1987)551; Z. Physik C39(1988)479.

[15] F.A. Berends, G. Burgers, W.L. van Neerven, Nucl. Phys. B297(1988)429;

E: ibid. B304(1988)921.

[16] F.A. Berends et al., Z Line Shape, in [2] vol 1, p.89.

[17] D. Bardin et al., Phys. Letters B229(1989)405. 
[18] W. Beenakker, F.A. Berends, W.L. van Neerven, in Proc. Int . Workshop on Radiative Corrections for $\mathrm{e}^{+} \mathrm{e}^{-}$Collisions, Schloss Ringberg, Tegernsee, FRG, April 1989, ed. J.H . Kühn, Springer-Verlag Berlin 1989, p.3.

[19] M. Böhm., W. Hollik et al., Forward-Backward Asymmetries, in [2] vol 1, p. 203.

[20] E.A. Kuraev, G.V. Meledin, Nucl. Phys. B122(1977)485.

[21] G. Passarino, Nucl. Phys. B204(1982)237.

[22] M. Igarashi, N. Nakazawa, T. Shimada, Y. Shimizu, Nucl. Phys. B263(1986)347.

[23] O. M. Fedorenko, T. Riemann, Acta Phys. Polonica B18(1987)761.

[24] M. Bilenky, A. Sazonov, JINR Dubna prepr. E2-89-792.

[25] B. Adeva et al., L3 Collab., Phys. Letters B236(1990)109, Phys. Letters B238(1990)122.

[26] D. Bardin, O. Fedorenko, T. Riemann, in Proc. Int. Symp. on Special Topics in Gauge Field Theories, Ahrenshoop, GDR, Nov. 1985, ed. F. Kaschluhn, Berlin-Zeuthen prepr. PHE 85-15 (1985), p. 228; ibid., Oct. 1986, PHE 86-13 (1986), p. 334.

[27] A. Akhundov, D. Bardin, T. Riemann, Nucl. Phys. B276 (1986)1;

W. Beenakker, W. Hollik, Z.Physik C40(1988)141.

[28] F.A. Berends, G. Burgers, W. Hollik, W.L. van Neerven, Phys. Letters B203(1988)177.

[29] D. Bardin et al., Z. Physik C44(1989)493; Comput. Phys. Commun. 29(1989)185.

[30] W. Hollik, prepr. DESY 88-188(1988), in: Fortschr. Physik 38 (1990) 165.

[31] D. Bardin, T. Riemann, Zeuthen prepr. PHE 89-11, to appear in 40].

[32] H.S. Strubbe, Comput. Phys. Commun. 8(1974)1.

[33] A.Hearn, Reduce Users Manual, Rand Public. CP78(Rev.7/87).

[34] D. Bardin, P. Christova, O. Fedorenko, Nucl. Phys. B175(1980)435.

[35] D. Bardin, N. Shumeiko, Nucl. Phys. B127(1977)242.

[36] A. Akhundov, D. Bardin, O. Fedorenko, T. Riemann, JINR Dubna prepr. E2-84-777(1984); E2-87-664(1987).

[37] D. Bardin, C. Burdik, P. Christova, T. Riemann, Z. Physik C42(1989)679.

[38] D. Bardin et al., Z-BIZON package, L3 note 679(Sept.1989), unpublished, see also [3].

[39] M. Bilenky, M.Sachwitz, Zeuthen prepr. PHE 89-12, to appear in 40].

[40] N. Dombey (ed.), Proc. Research Workshop on Radiative Corrections: Results and Perspectives, Univ. of Sussex, Brighton, July 1989, to appear.

[41] S. Jadach, J.H. Kuehn, R. Stuart, Z. Was, Z. Physik C38(1988)609.

[42] S. Jadach, Z. Was, preprint MPI-PAE/PTh 33/89; Phys. Letters B219(1989)103;

T.Riemann,Z. Was, Mod. Phys. Letters A4(1989)2487. 
[43] F. Aversa, M. Greco, Phys. Letters B228(1989)134.

[44] M. Greco, LNF Frascati prepr. LNF-89/042(1989); LNF-89/069(PT)(1989), invited talk presented at the Workshops "QED Structure Functios" - Ann Arbor (May 1989) and "Radiative Corrections: Results and Perspectives" Brighton (July 1989);

M. Greco, O. Nicrosini, Phys. Letters B240(1990)219.

[45] M. Greco, G. Pancheri-Srivastava, Y. Srivastava, Phys. Letters B56(1975)367; G. Altarelli, R. K. Ellis, R. Petronzio, Nuovo Cim. Letters 13(1975)393; A. Kraemmer, B. Lautrup, Nucl. Phys. B95(1975)380.

[46] J. Jersak, E. Laermann, P. M. Zerwas, Phys. Rev. D25 (1982)1218. 